\title{
O Pensamento Computacional presente na Resolução de Problemas Investigativos de Matemática na Escola Básica
}

\author{
Rafaela da Silva Bobsin ${ }^{1}$, Natália Bernardo Nunes ${ }^{1}$, Anelise Lemke Kologeski ${ }^{1}$, Aline \\ Silva de Bona ${ }^{1}$ \\ ${ }^{1}$ Instituto Federal de Educação, Ciência e Tecnologia do Rio Grande do Sul - Campus \\ Osório \\ Av. Santos Dumont, 2127, Albatroz, Osório/RS \\ rafaela.bobsin03egmail.com, nataliabernunesegmail.com, \\ anelise.kologeskidosorio.ifrs.edu.br, aline.bonalosorio.ifrs.edu.br
}

\begin{abstract}
Considering the lack of digital inclusion in the regular school, this work reports the activities developed in an extension project, applied in basic schools, which aims to contextualize Mathematics with Computational Thinking, through workshops that work with investigative problems resolution, sharing here the qualitative and quantitative analysis of 2 specific problems. The methodology is a theoretical study and a case study. The results shown a clear improvement of the students in the proposed activities, reaching approximately 38\% of improvement, with the appropriation of the Computational Thinking linked to the investigative problems resolution, closely related to specific tasks from Mathematics discipline.
\end{abstract}

Resumo. Considerando a falta de inclusão digital no Ensino Básico, este trabalho relata as atividades desenvolvidas em um projeto de extensão, aplicado em escolas básicas, que objetiva contextualizar a Matemática com o Pensamento Computacional, por meio de oficinas que trabalham com a resolução de problemas investigativos, compartilhando aqui a análise qualitativa e quantitativa de 2 problemas específicos. A metodologia é um estudo teórico e um estudo de caso. Os resultados mostram um nítido aproveitamento dos alunos nas atividades propostas, chegando a aproximadamente $38 \%$ de melhoria, com a devida apropriação do Pensamento Computacional atrelada a resolução de problemas investigativos, intimamente relacionada com tarefas específicas da disciplina de Matemática.

\section{Introdução}

Atualmente é comum os estudantes apresentarem um desinteresse em atividades tradicionais de sala de aula. Com essa problemática, ocorre a redução do envolvimento para a realização das mesmas e do interesse dos professores em estudar e procurar por novas metodologias que despertem o interesse dos educandos. Em Matemática, particularmente, existem tendências e metodologias para a sala de aula, atreladas a diferentes recursos e estratégias, conforme Bona (2012), Fiorentini e Lorenzato (2009).

A resolução de problemas de Matemática, que é um cenário usual e natural nessa ciência, está se relacionando cada vez mais a diferentes recursos tecnológicos. Contudo, vem percebendo-se que o essencial não é o recurso, segundo Bona (2016), mas a forma e o meio escolhido pelo professor para explorar a resolução dos problemas, como é o caso dos problemas investigativos a serem apresentados nesse trabalho. Eles proporcionam uma 
atividade lúdica, diversificada e interessante aos estudantes, e paralelamente permitem a resolução coletiva, com seus pares, permitindo aos estudantes que pensem juntos, fazendo, compreendendo e trocando ideias, escrevendo ou registrando apenas uma solução, ou cada um da sua maneira, conforme Bona (2012).

Além dos problemas investigativos, um elemento atrativo aos estudantes são as sequências lógicas para os problemas que são próximos da realidade de cada um, como por exemplo: o passo a passo para construir uma referência a uma pesquisa da escola, ou compreender como pagar uma conta digital em um site seguro, gerando o comprovante, inclusive. Tal sequência é muito utilizada na Matemática, assim como na Informática, como defende Bona (2012), e então se percebe o quanto o Pensamento Computacional está presente nas resoluções de problemas investigativos, e que podem ser gradualmente exploradas. Outro elemento importante é a linguagem, pois cada estudante organiza uma forma de escrever sua resolução, e que nem sempre faz uso da linguagem Matemática, mas usa uma organização de pensamento que é muito importante para a Informática, como ao construir um algoritmo, por exemplo, adotando posteriormente uma linguagem de programação específica.

O trabalho de Araújo et. al. (2018) destaca o quanto é atrativo aos estudantes compreender de forma lúdica, por meio de jogos, o Pensamento Computacional, e que este contempla a necessidade de compreensão de uma sequência lógica, bem como a organização dos pensamentos em blocos, sendo que nessa ação está implícita a abstração, a automação e a análise do seu próprio pensamento através da resolução proposta. Outro trabalho que colabora com a ideia de explorar problemas do tipo que o estudante possa criar suas hipóteses, testar, reconstruir, e novamente tentar validar com ajustes, segundo seus conhecimentos, para a aprendizagem de Matemática, é o trabalho de Leitão e Castro (2018), com o uso da plataforma Scratch. Ambos os trabalhos contemplam a importância de que os problemas atrelados a uma lógica, baseada em tecnologias, fazem parte da vida cotidiana, e que se faz necessário aprender a resolver além disso, pois a escola não deve delimitar um tipo de problema para explorar um conceito ou a criatividade dos estudantes.

Assim, este trabalho tem como pergunta de pesquisa: Como atrelar o Pensamento Computacional na Escola Básica? E objetiva explorar conceitos de Matemática e recursos tecnológicos utilizados na vida cotidiana, através de problemas investigativos de Matemática montados de forma a serem atrativos, investigando o potencial do Pensamento Computacional nas aulas de Matemática da Escola Básica.

Para tornar viável tal aplicação, um projeto de extensão foi criado para estimular o Pensamento Computacional aliado aos problemas investigativos da Matemática, com a participação majoritária de alunos de $9^{\circ}$ ano do Ensino Fundamental. Para isso, atividades lúdicas foram planejadas e aplicadas com o uso de ferramentas da Tecnologia da Informação e Comunicação, em oficinas, levando a inclusão digital para mais perto dos participantes, oportunizando um momento de aprendizado, autonomia e estímulo ao desenvolvimento.

\section{Problemas Investigativos na Matemática}

Fazer uso de problemas em Matemática que sigam uma metodologia, ou seja, contextualizados, aproxima a disciplina das rotinas da vida pessoal e profissional. Essa metodologia, quando atrelada a ação de investigar, leva em consideração os conhecimentos dos estudantes e suas percepções, hipóteses e compreensões sobre o problema a ser revolvido. Se prioriza a autonomia dos estudantes, porque para validar suas hipóteses, será necessário pesquisar e encontrar meios e formas para tal e saber argumentar com os colegas para ampla 
compreensão. Nessa cadeia de pensamentos e do processo de fazer e compreender, o estudante desenvolve habilidades sequenciais e lógicas, além dos conceitos necessários para solucionar o problema [Bona, 2016].

A ação de investigar, para Ponte, Brocardo e Oliveira (2006), é procurar conhecer o que não se sabe, é descobrir relações entre objetos Matemáticos conhecidos ou desconhecidos de maneira a procurar identificar suas propriedades e o processo de criação, e contempla quatro passos: (1) identificar o problema a resolver; (2) formular hipóteses; (3) realizar testes; e (4) argumentar/validar/provar, seja sozinho ou em grupo. Ainda, segundo Bona e Souza (2015), a ação de investigar implica em compreender e buscar soluções justificadas para os problemas, e então contemplam elementos como: mobilizar os estudantes a aprender; a aprendizagem decorre da ação dos estudantes; a prática docente baseada no diálogo e criatividade dos estudantes, pelo fato único de que cada estudante terá uma lógica de pensamento, e compreender essa multiplicidade é potencializar a capacidade de aprender.

Ao resolver um problema, se faz uso de representações, sejam escritas, desenhadas ou gráficas. Assim como a Matemática tem uma forma de se comunicar com sua linguagem simbólica, a Informática também tem, segundo os Parâmetros Curriculares Nacionais de Matemática [PCN, 1999], e ao se pensar em Pensamento Computacional implicitamente se contempla uma ou mais formas de representar a mesma solução do problema, devido a diferentes pessoas que solucionam e aos diferentes recursos tecnológicos explorados.

Cabe destacar que a ação investigativa atrelada às tecnologias digitais proporciona aos estudantes uma interatividade [Bona 2016], e que segundo Bona (2012) maximiza o processo de aprendizagem pelo fato das interações serem além de colaborativas, mas cooperativas, no momento em que se realiza o passo a passo em conjunto, e então, a sequência lógica da resolução de problemas investigativos de Matemática se aproxima do Pensamento Computacional, segundo a variedade de contextos que se fazem necessários [Fiorentini e Lorenzado 2007] [D’Ambrosio 1996].

\section{Pensamento Computacional}

Para Silva (2019) a Matemática contempla o Pensamento Computacional, que por sua vez, segundo Wing (2010), é compreendido como o método, a maneira, a forma de pensar presente na abstração/lógica de um problema, bem como suas possíveis resoluções de modo que estas sejam representadas de uma forma que pode ser testada, executada, logicamente por um processador, ancorada em artefatos computacionais.

Considerando a disciplina de Matemática na Escola Básica, o objetivo torna-se desenvolver habilidades como lógica sequencial, a construção de ideias generalizadas, como os algoritmos das operações com números reais, interpretar e representar, além das construções, segundo o PCN [PCN, 1999] e a Base Nacional Comum Curricular [BNCC, 2018]. Com isso, torna-se evidente que a fusão entre o Pensamento Computacional e a Matemática não se trata de qualquer atividade de Matemática, mas de atividades do tipo problemas investigativos, que podem contemplar (mas não apenas): lista de problemas, projetos interdisciplinares, projetos de pesquisa, demonstrações, aplicações em outras áreas do conhecimento, como jogos ou atividades onde segue-se um padrão, onde o estudante possa se identificar, explorar e propor uma solução por ele testada e válida. Atividades do tipo "siga o modelo", que expressam uma mera cópia e pouca construção dos estudantes, com pouca abstração, não contemplam a proposta deste trabalho e não permitem atrelar a autonomia, a 
aprendizagem e o desenvolvimento do aluno aos recursos propostos pela relação entre o Pensamento Computacional e a Matemática.

Relatos de autores comprovam que a relação entre o Pensamento Computacional e a Matemática está interligada, e pode facilitar a atuação docente no desenvolvimento das atividades, pois facilita o domínio em sua aplicação, bem como a integração das atividades com o currículo existente e as vivências cotidianas dos estudantes [Marques et. al. 2017]. Riboldi e Reichert (2019) mostram como o uso da plataforma Scratch pode auxiliar no ensino de funções matemáticas e no uso do Pensamento Computacional em sala de aula, proporcionando um momento mais dinâmico e interessante aos alunos. Os autores mostram como o uso dessa ferramenta vai de encontro a BNCC [BNCC, 2018] e como o entendimento dos participantes sobre os conceitos abordados melhorou significativamente ao fim das atividades realizadas. Já no trabalho de Leitão e Castro (2018), é também relatado o uso do Scratch, mas em uma oficina para docentes e futuros profissionais que serve para desenvolver novos conceitos a serem utilizados nas aulas de Matemática, desenvolvendo um raciocínio a fim de passar o sistema de ensino instrucionista para um de aprendizagem autoral com esta plataforma, já que, segundo os resultados apresentados pelo autor, a cada tentativa, maior é o desenvolvimento de habilidades com o Pensamento Computacional, que por sua vez auxilia na resolução de problemas da disciplina de Matemática.

Contudo, o Pensamento Computacional é, por enquanto, um conteúdo escolar não foi formalizado como disciplina dentro dos currículos da Escola Básica, embora esse processo de curricularização esteja se tornando alvo de discussões entre os membros da comunidade brasileira de Informática na Educação. Por isso, como já dito anteriormente, a ideia deste trabalho consiste em atrelar os problemas investigativos de Matemática ao Pensamento Computacional para que isso seja viável dentro das escolas.

\section{Metodologia}

As oficinas propostas visam estimular o desenvolvimento do Pensamento Computacional, trabalhando com aspectos da Matemática e da Programação Básica, além da interpretação de texto e do raciocínio lógico, contemplando a metodologia de Aprendizagem Baseada na Resolução de Problemas, mencionada por Schuhmacher et al. (2016). As atividades de Pensamento Computacional são divididas em:

- Desplugadas: utilizam apenas atividades concretas sem o uso do computador. Normalmente, ela ocorre na própria escola, especialmente se não há um laboratório de informática disponível. São exemplos de atividades realizadas os jogos "Estacionamento Algorítmico" e a "Programação com Papel Quadriculado", sendo esta última relacionada ao Problema Investigativo 1 apresentado na Figura 1 . Em ambas as atividades, os participantes precisam desenvolver ou seguir algoritmos para realizar o que é solicitado.

- Plugadas: as atividades fazem uso de recursos digitais, ou seja, necessitam do uso de computadores. Diferentes jogos como "A Fazendeira" ${ }^{3}$ e o "Labirinto Clássico" 4 são apresentados aos alunos, utilizando a programação em blocos para a devida conclusão das tarefas, sendo o Problema Investigativo 2 oriundo deste último, conforme apresentado na Figura 2. Os jogos apresentam diferentes níveis de dificuldade, permitindo uma evolução

\footnotetext{
1 Disponível em: http://computacional.com.br/

2 Disponível em: https://studio.code.org/s/20-hour/stage/4/puzzle/1

3 Disponível em: https://studio.code.org/s/20-hour/stage/9/puzzle/1

4 Disponível em: https://studio.code.org/hoc/1
} 
gradual ao aluno. Normalmente, essas oficinas ocorrem nas dependências da nossa instituição, oportunizando aos participantes conhecerem as atividades e os espaços oferecidos por ela, além das tarefas realizadas durante a execução da oficina.

Essa divisão das oficinas acontece para atender as necessidades específicas de cada escola, já que nem todas possuem laboratórios de informática ou disponibilidade de deslocamento até a nossa instituição, permitindo assim que elas optem pela realização de apenas uma das oficinas, caso necessário. Cada oficina tem a duração de até 4 horas, conforme disponibilidade das escolas, e é aplicada por pelo menos 2 membros da equipe de execução do projeto (composta por 6 alunos bolsistas, 2 professoras e um técnico em Tecnologia da Informação). As oficinas sempre são precedidas por um Pré-Teste, que avalia o conhecimento prévio dos participantes, e finalizadas com um Pós-Teste, que serve para comparar se houve alguma evolução no aprendizado deles após a realização das tarefas propostas. Ambos os testes utilizados em cada oficina são compostos por 5 questões, planejadas de acordo com as atividades aplicadas, e são idênticos entre o Pré e o Pós-Teste de cada oficina, para que os alunos possam observar seus erros respondendo corretamente as questões ao final da oficina. Os Problemas Investigativos 1 e 2 analisados neste trabalho são, respectivamente, questões trabalhadas nos Testes das oficinas Desplugadas e Plugadas, e são discutidos mais detalhadamente a seguir.

\subsection{Contexto dos Problemas Escolhidos}

Inicialmente, as oficinas foram idealizadas como uma forma lúdica de levar a inclusão digital aos estudantes de escolas de Ensino Fundamental. Por isso, foram escolhidos diversos problemas investigativos para serem trabalhados em sala de aula, de forma dinâmica e autônoma, em grupos, e que fizessem os alunos refletir sobre a importância da compreensão de determinados conceitos da Computação atrelados ao Pensamento Computacional, que estão presentes diariamente em nossas mais diversas atividades do cotidiano. Por isso, o Problema Investigativo 1, que consta na Figura 1, foi planejado para apresentar aos estudantes, de forma desplugada, a ideia de seguir instruções bem definidas para a obtenção de um objetivo, como em um algoritmo, enquanto que o Problema Investigativo 2, que consta na Figura 2, traz o exemplo de jogo digital cujo as instruções precisam ser devidamente organizadas para a conclusão da fase do jogo.

PROBLEMA INVESTIGATIVO 1. Considere os 4 comandos e a grade $4 x 4$ apresentados abaixo. Qual a sequência de comandos correta para que a segunda coluna da grade fique completamente preenchida, de acordo com a programação no papel quadriculado? Considere o início da atividade no primeiro quadrado superior da esquerda.

$\rightarrow$ Mover para a direita. $\downarrow$ Mover para baixo.

—Mover para a esquerda. M Preencher quadrado.

a) $\rightarrow M \rightarrow M \rightarrow M \rightarrow M$

b) $\rightarrow M \downarrow M \downarrow M \downarrow M$

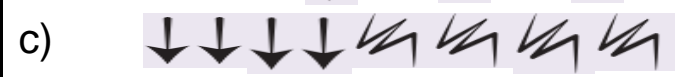

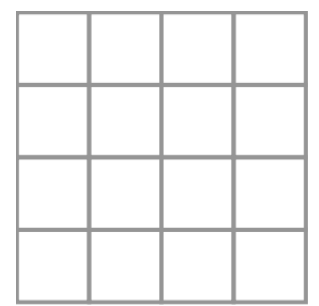

Figura 1. Problema Investigativo 1 baseado na Plataforma Code.org 
PROBLEMA INVESTIGATIVO 2. Levando em consideração a imagem à esquerda, qual bloco de comando que está faltando e deve ser introduzido na imagem à direita?
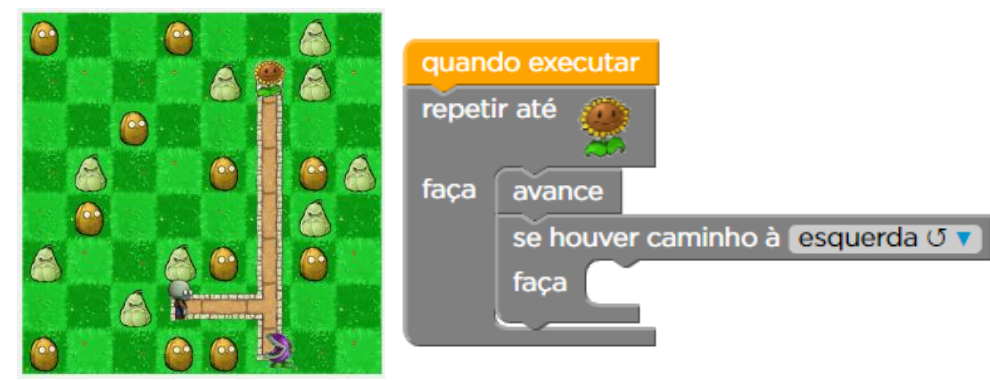

a) avance

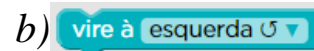

c)

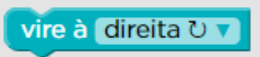

Figura 2. Problema Investigativo 2 baseado na Plataforma Code.org

No entanto, o leitor pode se perguntar: qual a relação desses problemas com a vida cotidiana? E a resposta é simples: passos são seguidos a todo momento por quem segue uma receita de bolo, as orientações de localização de um GPS ou por quem organiza tarefas em uma empresa. Sendo assim, o objetivo das atividades realizadas é que o estudante se dê conta de que o Pensamento Computacional nada mais é do que organizar devidamente as tarefas do cotidiano, para sua execução, sem o uso de recursos digitais necessariamente. Outros diversos problemas investigativos foram também utilizados durante as oficinas. Mas por questões de limitação de espaço, decidiu-se apresentar apenas dois deles neste trabalho, para uma reflexão mais aprofundada sobre eles conforme segue, onde são atrelados ao uso da Matemática em nosso contexto diário.

\subsection{Análise do Problema Investigativo 1 (Oficina Desplugada)}

Para solucionar o Problema Investigativo 1 é preciso observar, na Figura 1, a quantidade exata de comandos necessários para que a segunda coluna seja pintada, conforme as instruções fornecidas. Assim, o aluno precisa interpretar de forma matemática o caso, para saber a quantidade de instruções necessárias para executar até concluir o que é solicitado (preencher a segunda coluna da grade). Junto a isso, ele precisa compreender que o ponto de início é o mesmo para todos: o primeiro quadrado superior a esquerda. Sendo assim, a ordem dos comandos também importa para a obtenção do resultado. Desta forma, fica evidente a relação da resolução do problema com a sua generalização, pois não há nada concreto para apegar-se, exceto por uma figura no papel, e assim entender a localização, os passos, o sentido e a sequência de ações necessárias, evidenciando a necessidade de compreensão e interpretação de um algoritmo, para que o problema permita formalizar a compreensão do Pensamento Computacional, organizado em passos bem definidos.

Matematicamente pode-se resolver por matrizes, dando a resposta em termos que relacionam linha e coluna, e consequentemente daí surge a ação, como fazer o passo a passo para a resolução do problema, exigindo abstração e raciocínio do estudante, pois este precisa exatidão na sequência e saber onde começar. Diante das opções de resposta apresentadas para o aluno, duas saem completamente fora do escopo esperado, pois uma delas permanece na primeira linha da grade, enquanto a outra permanece na primeira coluna. Para que o aluno se encaminhe para a resposta correta, ele precisa identificar que há um passo necessário para atingir a segunda coluna, através do comando de movimentação dentro da linha, para a esquerda. Somente aí é que ele poderá pensar em como conciliar as instruções para preencher os quadrados e movimentar-se dentro da coluna, utilizando a Matemática sem precisar da 
realização de muito cálculo, fazendo uso de uma operação aritmética simples, em vez de apoiar-se no complexo uso da Álgebra ou da Geometria, por exemplo.

Para contextualizar a validação do Problema Investigativo 1, uma análise gradativa com estudantes participantes das oficinas foi realizada, por meio dos depoimentos dos mesmos. Como exemplificação, abaixo constam os depoimentos de dois alunos de Ensino Fundamental, que foram convidados para realização de uma resolução qualitativa:

- Análise do aluno 1 ( $8^{\circ}$ ano): Seguindo o enunciado, começo pelo primeiro quadrado superior. Preciso avançar o primeiro quadrado, à direita, e em seguida pintar, depois descer e pintar consecutivamente, até chegar ao último quadrado. Por isso é a opção " $b$ ".

- Análise do aluno 2 ( $9^{\circ}$ ano): Preciso de 8 ações para concluir, e essas ações precisam mudar de linha, não todas junto. Então encontro a resposta por exclusão. Se tiver de justificar, começando de baixo para cima ou de cima para baixo, ou pelos lados, temos que dobrar, pintar, descer, pintar, e assim sucessivamente mais 2 vezes para os dois últimos passos, totalizando 8 passos. Isso pensando no mínimo de passos, pois se puder ter mais passos, podemos ter muitos caminhos, que nem aparecem nas alternativas.

\subsection{Análise do Problema Investigativo 2 (Oficina Plugada)}

Espera-se que o estudante observe, na Figura 2, que não precisa saber a quantidade certa de passos necessários para o Zumbi atingir a Flor. Porém ele precisa perceber que existe um teste ("se houver caminho à") sendo realizado a cada passo efetuado, podendo então mudar o rumo do Zumbi. Tais elementos tornam o processo de resolução mais abstrato, porque o estudante precisa imaginar os movimentos que irão resolver o problema, sem quantificar, explorando a lógica sequencial, e também o sistema posicional (direção, sentido, ponto referencial) como o plano cartesiano, conforme achar necessário.

Por exemplo, se desejar, o aluno pode pensar no Zumbi, que está na posição $(0,0)$, andando no eixo X. Então ele anda (A,0), vira $90^{\circ}$ para a esquerda (A,B), sendo que no eixo de A ele ficou parado na horizontal, e apenas subiu em B para chegar na Flor, exigindo um deslocamento no eixo Y (A,C). Assim a ideia do problema é a lógica, e não necessariamente o cálculo da distância que foi percorrida, por exemplo, fazendo uso da lógica Matemática e sequencial, construindo habilidades para o Pensamento Computacional através do uso de funções de recursos, exigindo abstração.

Nesse problema, fica evidente os passos da resolução de um problema investigativo, pois há uma exploração inicial, seguida pela formulação de hipóteses, pelos testes das hipóteses e pela validação, quando o aluno encontra de fato a alternativa com lógica. Paralelamente, relacionando ao Pensamento Computacional, se o estudante não compreende a imagem de comandos e sua inserção hierárquica de execução, ele não consegue nem criar a hipótese, então ora a Matemática está apoiando o Pensamento Computacional e vice-versa, embora tudo dependa sempre do referencial do estudante quanto ao processo de abstração para a devida resolução do problema.

$\mathrm{Na}$ validação do Problema Investigativo 2, abaixo constam, como exemplos, os depoimentos dos mesmos dois estudantes mencionados na seção 4.2:

- Análise do aluno 1 ( $8^{\circ}$ ano): Como o comando manda avançar até ter um caminho, e se não tiver ele irá virar à esquerda, o próximo comando é avançar. Opção " $a$ ”.

- Análise do aluno 2 ( $9^{\circ}$ ano): Tenho que andar dois passos de um quadrado, daí virar à esquerda se estiver de frente inicialmente, e ando mais 8 passos para chegar na flor. $\mathrm{E}$ 
logicamente preciso virar à esquerda. O caminho é muito curto para pensar em repetição, mas entendo a função pois ela aparece num jogo do celular, e é importante pois executa mais rápido.

\section{Análise dos Dados}

Desde o ano de 2017 o projeto já atendeu mais de 440 alunos da rede pública de ensino. Contudo, decidimos apresentar um estudo de caso com as turmas do ano de 2019, que trabalharam efetivamente com os problemas investigativos apresentados, totalizando 237 alunos, majoritariamente do $9^{\circ}$ ano do Ensino Fundamental de escolas públicas municipais e estaduais, dos quais 169 deles participaram das Oficinas Desplugadas, e 169 das Oficinas Plugadas, sendo que alguns destes participantes tiveram a oportunidade de participação nas duas oficinas. Com isso, pretende-se detalhar e apontar reflexões sobre as questões abordadas. Diversos problemas similares foram apresentados durante as oficinas, mas por questões de limitação, apenas 2 deles foram escolhidos para serem apresentados neste trabalho. Na Tabela 1 apresenta-se quantitativamente o percentual ${ }^{5}$ dos estudantes que acertaram os problemas antes e depois das oficinas, bem como a amostragem considerada.

Tabela 1. Resultados obtidos no Pré e no Pós-Teste das oficinas

\begin{tabular}{|c|c|c|c|}
\hline & Acertos Pré & Acertos Pós & Amostragem \\
\hline Problema Investigativo 1 - Oficina Desplugada & $63,31 \%$ & $83,43 \%$ & 169 alunos \\
\hline Problema Investigativo 2 - Oficina Plugada & $17,16 \%$ & $55,03 \%$ & 169 alunos \\
\hline
\end{tabular}

O Problema Investigativo 1 aborda uma situação mais próxima do real e do concreto, e é possível perceber que os alunos conseguem desenvolvê-lo mais facilmente, melhorando em até $19,88 \%$ a compreensão e resolução dele após a realização da oficina, sendo que o resultado obtido no Pré-Teste já é bastante satisfatório (63,31\% de acertos). Já o Problema Investigativo 2 relaciona conceitos mais abstratos, que fazem uso de Programação Básica, exigindo uma maior capacidade de compreensão do estudante. Por isso, no Pré-Teste, o resultado obtido foi de apenas $17,16 \%$ de acertos, pois foi o primeiro contato de muitos estudantes com a organização de comandos e instruções em formato de um algoritmo específico de programação. Após o término da oficina, observou-se um progresso nos resultados de até 37,87\%, mostrando assim que o nível de dificuldade e abstração necessário foi satisfatoriamente atingido por muitos alunos, aumentando consideravelmente para a devida compreensão e resolução da atividade após a realização da oficina oferecida. Contudo, para elevar o número de acertos além de 55,03\%, acreditamos na necessidade de mais encontros e mais horas de atividades, para que todos alunos possam atingir o mesmo nível de rendimento nas oficinas oferecidas, já que alguns alunos apresentaram maiores dificuldades no desenvolvimento das atividades propostas.

\section{Considerações Finais}

Relacionar a resolução de problemas investigativos de Matemática com o uso do Pensamento Computacional em sala de aula permite uma prática docente que visa trabalhar a autonomia do estudante, preparando-o para o futuro, responsabilizando-o também pelo seu próprio aprendizado, mobilizando nele o ato de aprender.

5 Cálculo do percentual: dividir o número de acertos pelo número de alunos em cada oficina. 
O ato de aprender na Escola Básica "o que é investigar" através da Matemática abre portas para entender essa modalidade de pensar em outras áreas do conhecimento, e também da aplicação em outros contextos da área das Ciências Exatas. Uma vez que o estudante entenda as sequências, representações, organizações, e suas conceituações atreladas, constrói-se um processo denso de abstrações, e a generalização e reciprocidade é o próximo passo do processo de desenvolvimento e aprendizagem, e que mobiliza no estudante um processo de reflexão para a vida pessoal e profissional. Assim, pode-se fazer um paralelo: depois de tais reflexões, o estudante não precisa mais da ajuda de um tutorial para buscar determinada solução, mas sim buscá-la por meio do uso da lógica, sendo ele próprio capaz de conquistar o resultado através do raciocínio lógico e de passos bem definidos, atrelados ao Pensamento Computacional, e não apenas de forma meramente intuitiva.

Com os problemas abordados nas oficinas, foi possível observar que para o aluno, explorar o concreto é mais fácil, ajudando a organizar as ideias, e a estabelecer as operações lógicas, enquanto que a compreensão de problemas mais abstratos, demanda mais tempo e maior complexidade aos estudantes, não sendo uma transição de simples. Isso pode ser observado nos resultados obtidos, onde houve um progresso de 19,88\% dos estudantes que conseguiram acertar o Problema Investigativo 1, enquanto houve 37,87\% a mais de acertos para Problema Investigativo 2, após o término das oficinas realizadas, sendo o último de maior complexidade, exigindo maior abstração. Portanto, conclui-se a importância da valorização dos problemas investigativos pelos docentes de Matemática, pensando além de trabalhar o Pensamento Computacional diretamente, para que ao longo da vida do estudante, e em oportunidades como esse projeto de extensão, o estudante perceba habilidades e competências que podem ser atreladas aos mais diferentes contextos, servindo como portas para o futuro, independente da área do conhecimento. E tem-se como desafio futuro, em processo, a criação de um conjunto de atividades desplugadas e plugadas, inclusive de uma metodologia, inovadoras para a inserção do Pensamento Computacional na Escola Básica através da disciplina de Matemática, por meio da resolução de problemas investigações, seja como pesquisa e também extensão.

\section{Referências}

Araújo, L.; Silveira, H. U. C. ; Mattos, M. (2018) Ensino do pensamento computacional em escola pública por meio de uma plataforma lúdica. Anais dos Workshops do Congresso Brasileiro de Informática na Educação, p. 589. ISSN 2316-8889. Disponível em: $<$ https://www.br-ie.org/pub/index.php/wcbie/article/view/8284>. Acesso em: 02 jul. 2020. doi:http://dx.doi.org/10.5753/cbie.wcbie.2018.589.

BNCC (2018). Ministério da Educação; Secretaria de Educação Básica; Conselho Nacional de Educação. Base Nacional Comum Curricular: educação é a base. Brasília: MEC; SEB; CNE; 2018. Disponível em: <http://basenacionalcomum.mec.gov.br/a-base $>$. Acesso em: 22 jun. 2020.

Bona, A. S. (2012) Espaço de Aprendizagem Digital da Matemática: o aprender a aprender por cooperação. Tese (Doutorado). Programa de Pós-Graduação em Informática na Educação. Porto Alegre: UFRGS.

Bona, A. S. (2016) Aulas Investigativas e a construção de conceitos de matemática: um estudo a partir da teoria de Piaget. Curitiba: CRV.

Bona, A. S.; Souza, M. T. C. C. (2015) Aulas investigativas e a construção de conceitos de matemática: um estudo a partir da teoria de Piaget. Revista Psicologia USP, v.26, n.2, p. 
240-248. Disponível em: < https://www.scielo.br/pdf/pusp/v26n2/0103-6564-pusp-26-0200240.pdf $>$. Acesso em: 17 jun. 20.

D'Ambrosio, U. (2016) Educação Matemática: da teoria a práxis. Coleção Perspectivas em Educação Matemática. Campinas, SP: Papirus.

Fiorentini, D.; Lorenzato, S. (2017) Investigação em educação matemática: percursos teóricos e metodológicos. Campinas: Autores Associados.

Leitão, D.; Castro, J. B. (2018) Construção de Recursos Digitais de Matemática: uma experiência de autoria com o Scratch. Anais dos Workshops do Congresso Brasileiro de Informática na Educação, p. 510. ISSN 2316-8889. Disponível em: <https://www.brie.org/pub/index.php/wcbie/article/view/8276>. Acesso em: 02 jul. 2020. doi:http://dx.doi.org/10.5753/cbie.wcbie.2018.510.

Marques, M.; Cavalheiro, S.; Foss, L.; Avila, C.; Bordini, A. (2017) Uma Proposta para o Desenvolvimento do Pensamento Computacional Integrado ao Ensino de Matemática. Brazilian Symposium on Computers in Education (Simpósio Brasileiro de Informática na Educação - SBIE), p. 314. ISSN 2316-6533. Disponível em: <https://brie.org/pub/index.php/sbie/article/view/7560/5356>. Acesso em: 02 jul. 2020. doi:http://dx.doi.org/10.5753/cbie.sbie.2017.314.

PCN (1999). Ministério da Educação; Secretaria de Educação Básica; Conselho Nacional de Educação. Parâmetros Nacionais da Educação: Ensino Médio. Brasília: MEC; SEMTEC; $4 \mathrm{v} ; 1999$.

Ponte, J. P.; Brocardo, J.; Oliveira, H. (2006) Investigações matemáticas na sala de aula. Belo Horizonte, MG: Autêntica.

Riboldi, S. M. O.; Reichert, J. T. (2019) A linguagem de programação Scratch e o ensino de funções: uma possibilidade. Anais do XXV Workshop de Informática na Educação (WIE 2019). Disponível em: <https://br-ie.org/pub/index.php/wie/article/view/8497>. Acesso em: 03 de julho de 2020.

Schuhmacher, E.; Ropelato, D.; Schuhmacher, V. (2016) O Desenvolvimento do Pensamento Computacional no Ensino Médio por meio de Ambientes de Programação. XIV International Conference on Engineering and Technology Education. Salvador/Brasil, p. 239-243.

SILVA, L. C. L. (2019) A relação do Pensamento Computacional com o ensino de Matemática na Educação Básica. Dissertação (mestrado) - Universidade Estadual Paulista (Unesp), Faculdade de Ciências e Tecnologia, Presidente Prudente. Disponível em: $<$

https://repositorio.unesp.br/bitstream/handle/11449/191251/silva_lcl_me_sjrp.pdf?seque

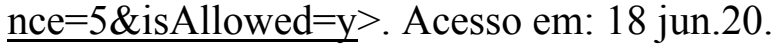

Wing, J. M. (2010) Computational thinking: what and why?. Disponível em: http://www.cs.cmu.edu/ CompThink/resources/TheLinkWing.pdf. Acesso em: 19 jun. 20. 